\title{
Violencia de género en mujeres Azogueñas
}

\section{Gender-based violence in women Quicksilver}

\author{
María de los Ángeles Estrella González ${ }^{* 1,2}$, Andrés Alexis Ramírez Coronel ${ }^{1}$, Rosa Elvira Minchala Urgilés ${ }^{1}$, Luis \\ Francisco Altamirano Cardenas ${ }^{1}$ \\ ${ }^{1}$ Universidad Católica de Cuenca, Ecuador \\ ${ }^{2}$ Universidad del Zulia, Maracaibo, Venezuela \\ *maria.estrella@ucacue.edu.ec
}

DOI: https://doi.org/10.26871/killkanasalud.v4i4.741

\begin{abstract}
Resumen
Contexto: La violencia de género es un tema que genera impacto a nivel mundial, además de ser un problema de salud pública, ya que existe cifras elevadas de violencia patrimonial, psicológica, física y sexual que suceden diariamente y que no son reportados por temor, omisión y justificación, es por ello que nace la importancia de realizar la presente investigación. Objetivo: Describir la violencia de género en mujeres que acuden al Centro de Salud de Azogues. Metodología: Estudio no experimental de tipo descriptivo, analítico, prospectivo y de corte trasversal con un enfoque cuantitativo. Los participantes fueron Mujeres de 15 a 49 años de edad que acuden a consulta externa. Se diseñó una encuesta que incluía factores sociodemográficos y constaba de 27 preguntas, que recogían datos sobre los tipos de violencia patrimonial, psicológica, física y sexual. Resultados: de 351 mujeres que conformaban la muestra establecida, en donde más del $50 \%$ pertenecía a la población de adulto joven, manifestaron violencia patrimonial 22,5\%, Psicológica 45,6\%, Física $20,8 \%$ y sexual el $13,1 \%$. Existiendo un nivel de significancia positiva entre la edad y la violencia patrimonial, psicológica y física, estado civil con todos los tipos de violencia y el nivel de instrucción con un nivel de significancia negativa con relaciona a la violencia patrimonial, psicológica y física.
\end{abstract}

Palabras clave: violencia de género, mujeres, consulta externa.

\begin{abstract}
Gender violence is an issue that generates worldwide impact, in addition to being a public health problem, since there are high levels of patrimonial, psychological, physical and sexual violence that occur daily and are not reported due to fear, omission and Justification, that is why the importance of carrying out this research is born. Describe gender violence in women who attend the Health Center of Azogues. Non-experimental study of descriptive, analytical, prospective and cross-sectional type with a quantitative approach. The participants were Women 15 to 49 years of age who went to an outpatient clinic. A survey was designed that included sociodemographic factors and consisted of 27 questions, which collected data on the types of patrimonial, psychological, physical and sexual violence. Results were obtained from 351 women who made up the established sample, where more than 50\% belonged to the young adult population, they showed patrimonial violence 22.5\%, Psychological 45.6\%, Physical 20.8\% and sexual 13, one\%. There is a level of positive significance between age and patrimonial, psychological and physical violence, marital status with all types of violence and the level of instruction with a level of negative significance related to patrimonial, psychological and physical violence.
\end{abstract}

Keywords: gender violence, women, external consultation .

\section{Introducción}

En el año de 1994 la Organización Panamericana de Salud (OPS), en la Declaración de la Conferencia Interamericana sobre Sociedad, Violencia y Salud reconoce a La Violencia como un problema que afecta a toda la población. En la Conferencia Internacional de Las Naciones Unidas Sobre Población y Desarrollo realizada en el Cairo, se reconoció que los derechos reproductivos son derechos humanos, que la violencia de género es un obstáculo a los derechos reproductivos de las mujeres, salud sexual, educación, participación y desarrollo. ${ }^{1}$
La violencia de género es considerada como un problema de Derechos Humanos y de Salud Pública, afectando principalmente a las mujeres de todo el mundo sin importar clase social, etnia, cultura, edad, escolaridad y niveles de atención de salud, generando un impacto en la sociedad. Es registrada por la sociedad que le da existencia, tolera y justifica a quien comete este tipo de vulneración, cuya definición se manifiesta como todo acto que provoca daño: patrimonial, psicológica, físico y sexual, atentando contra la calidad de vida y el entorno de la mujer. ${ }^{2}$ 
De acuerdo a Grossman ${ }^{3}$ la violencia de género es toda acción ejercida por una o varias personas, provocando de manera intencionada sufrimientos, maltratos y manipulaciones, atentando contra su dignidad.

Género es considerado un grupo de características culturales, políticas, jurídicas y económicas en donde la sociedad le atribuye lo que considera apropiado para hombres y mujeres. $^{4}$

El primer nivel de atención es la puerta de entrada a los servicios de salud, resuelve problemas y necesidades un $85 \%$, siendo el primer contacto que tiene el usuario con los profesionales de salud. ${ }^{5}$

Los tipos de violencia que abarca la presente investigación son: Violencia Patrimonial donde el agresor utiliza los mecanismos para privarle de dinero y bienes a la víctima; violencia psicológica implica la manipulación mediante amenazas y humillaciones, disminuyendo su autoestima; violencia física aquella que deja huellas, moretones, hematomas, lesiones y fracturas siendo visible ante las demás personas; violencia sexual vulneración sobre su sexualidad utilizando comportamientos íntimos y agresivos sin su consentimiento. $^{6}$

Para desencadenar cualquier tipo de violencia existe un ciclo que se detalla a continuación: fase de acumulación de tensión donde el agresor se muestra irritable e insoportable y cualquier actitud de la víctima le disgusta; fase de explosión violenta aquí empiezan las agresiones, amenazas con acabar con la vida de la mujer y su entorno; fase de conciliación el agresor pide disculpas y demuestra que ha cambiado y escala de violencia en la cual el maltratador se ganó la confianza de la afectada y empieza nuevamente el ciclo de violencia. ${ }^{7}$

Los factores de riesgo que pueden llevar a una mujer para ser víctima de violencia son: Menor grado de escolaridad, historia de maltrato familiar, bajo nivel socioeconómico, inmigrantes, abuso de alcohol y drogas, machismo, mayor tasa de alfabetización en los hombres. ${ }^{8}$

Según la Organización Mundial de la Salud (OMS) las consecuencias de la violencia son las siguientes: muerte ya que el $38 \%$ fueron asesinadas por sus parejas, lesiones $42 \%$, riesgo de abuso de alcohol, contraer enfermedades de transmisión sexual, depresión, enfermedades mentales, embarazos y abortos no deseados. ${ }^{9}$

Según la encuesta Nacional de Estadísticas y Censos (INEC), aplicada en el año 2011, la tasa de incidencia de la población femenina según escenarios de violencia en la provincia de Cañar son los siguientes: mujeres víctimas de abuso sexual antes de los 18 años con $86.4 \%$, haber sufrido violencia a lo largo de su vida con un $68.7 \%$ y violencia por parte de su expareja $56,3 \% .^{10}$

En Ecuador la violencia de género es un problema de estado , a pesar de que existen políticas, leyes que contribuyen a la erradicación sigue existiendo un alto índice de violencia; esto se evidencia en la Encuesta de Relaciones Familiares y Violencia de Género, el tipo de mayor porcentaje es la psicológica con un $53.9 \%$, física $38 \%$, patrimonial $35.3 \%$ y sexual $25.7 \%$, la misma que puede ser mayor por la pareja o expareja que por otras personas y en donde se muestra en mujeres divorciadas en un $85.4 \%$ y un $78 \%$ en mujeres separadas. ${ }^{11}$ En la actualidad los roles de género dentro de la sociedad se comportan de acuerdo a lo que nuestra cultura determina; los mismos que se aprenden y adquieren dentro del núcleo familiar y social. ${ }^{12}$

El objetivo principal fue describir la Violencia de género en mujeres 15 a 49 años que acuden al Centro de Salud de Azogues.

De manera que se formuló la siguiente hipótesis: Los tipos de violencia de género están relacionados directamente con los factores sociodemográficos (edad, procedencia, etnia, estado civil, ocupación, nivel instrucción. Para el primero y segundo objetivo no se plantearon hipótesis ya que es un estudio netamente descriptivo.

Un estudio realizado en Ecuador sobre Violencia de género revela datos que 6 de 10 mujeres han sufrido algún tipo de violencia debido a diversos factores que las hacen vulnerables; ya sea por padecer algún tipo de discapacidad, ser indígenas o por el nivel socioeconómico bajo. ${ }^{13}$

La violencia de género es un problema no identificado correctamente por la población y esta sería la primera barrera que se debe romper para resolverlo satisfactoriamente. Se debe sensibilizar para tomar conciencia y así poder percibir de manera precoz, cualquier tipo de violencia y detenerla a tiempo de manera que no se desencadene situaciones que ponen en riesgo la vida de la víctima. ${ }^{14}$

Razón por lo que se realiza la presente investigación en mujeres de 15 a 49 años mediante la revisión bibliográfica de varios estudios, evidenciando un porcentaje alto de violencia sobre todo en mujeres indígenas con un nivel socioeconómico bajo, baja escolaridad, víctimas de violencia en la infancia, inmigrantes y parejas que abusan de sustancias estupefacientes son susceptibles a ser víctimas de violencia.

Contribuirá a describir los tipos de violencia de género, en mujeres que acuden a consulta externa debido a que en nuestra localidad no existe documentación o registro que especifique la situación de violencia que sufren las mujeres que acuden al Centro de Salud de Azogues.

\section{Marco teórico o antecedentes}

El surgimiento de nuevas enfermedades impacta a hombres y mujeres de manera diferente, provocando situaciones de desigualdad en los diferentes grupos de la población, en especial a los llamados "grupos vulnerables". 5 En el caso de la violencia de género, posiblemente la mayor parte de casos permanezcan invisibles y solo se denuncie entre un $25-28 \%,{ }^{6}$ son muchos los factores socio culturales que influyen en la decisión de la mujer para denunciar o poner un alto a la violencia, la Pandemia no solo llegó a afectar a la salud biológica y psicológicos, pues afectó en los ámbitos económicos, laborales, sanitarios y otras instancias como el Judicial, un sistema poco eficiente para hacer frente a la violencia. 
La situación de la mujer se limita ante la posibilidad de denunciar, ¿será que no quiso contar o no cuenta con medios de comunicación oportunos? y si logró denunciar ¿cuál fue la respuesta de las diferentes entidades del estado, responsables de aplicar medidas de protección frente a la agresión?, pues las circunstancias actuales frente a la pandemia, se han convertido en un factor propiciante de episodios de opresión, incertidumbre y sumisión de la mujer ante la vulneración de derechos, actos que afecta no solo a la víctima, sino deja huellas en quienes están en el círculo de convivencia familiar.

Varios países, durante la pandemia del COVID-19, ante un contexto económico desfavorable han identificado escenarios poco confiables para aminorar la violencia de género, realidad que se ve afectado por el desempleo, la inestabilidad laboral y el rol productivo y reproductivo de la mujer en el hogar; en el caso de China en el segundo mes del año, se triplicó las denuncias por violencia de género; las llamadas de auxilio fueron en aumento en Colombia, México, Australia, Chipre y Estados Unidos (entre el $20 \%$ al $91 \%$ ), en Francia la intervención de la policía en hogares reportados con violencia superó el $30 \%$ en una semana, en el caso de España aparentemente disminuyó un $40 \%$ las denuncias desde que dio inició el confinamiento, dato que obliga a deducir, que muchas mujeres se ven limitadas a realizar la denuncia por presencia del agresor en el hogar. ${ }^{7}$

La Organización Mundial de la Salud (OMS), define a la violencia como "El uso intencional de la fuerza, o el poder físico, de hecho, o como amenaza, contra uno mismo, otra persona o un grupo o comunidad, que cause o tenga muchas probabilidades de causar lesiones, muerte, daños psicológicos, trastornos del desarrollo o privaciones". ${ }^{10}$

En Colombia, en los seis primeros meses del 2017, el número de casos por violencia de género llegaron a 1137, acentuada en la violencia física (697), seguida de la sexual (260), negligencia y abandono (114) y la psicológica en menor rango (260), las mujeres fueron más afectadas $(76,6 \%)$, quienes ubican su residencia en el área urbana $(78,89 \%){ }^{8}$

La violencia de género evidencia su afectación en las diversas etapas de la vida, estadísticas de las encuestas realizadas por el INEC 2019, 32 de cada 100 mujeres fueron víctimas de violencia: área urbana $33,3 \%$ y rural $27.4 \%$; casada o unida $28.7 \%$; la edad más afectada entre los 15 a 44 años, la escolaridad no es un limitante para la violencia, pues los datos avizoran la presencia de la violencia en los diferentes niveles y estratos sociales y culturales, ímpetu que muchas veces no se la rechaza, más bien se la acepta. ${ }^{9}$

Por lo tanto, la violencia de género es toda acción ejercida por una o varias personas, provocando de manera intencional maltratos, sufrimientos, presión, y manipulación, de forma que atente sobre la dignidad de la persona. ${ }^{11}$ Manifestada como una relación de poder, una situación de desigualdad e imposición del hombre hacia la mujer, ocasionando secuelas físicas, psicológicas, sexuales y patrimoniales, caracterizado por el uso de la fuerza, el chantaje y amenazas, esta asimetría de jerarquía vulnera los derechos, restringe la posibilidad de la mujer de tomar decisiones asertivas en su vida o la culminación de su proyecto de vida. $^{12}$

\section{Metodología}

\subsection{Tipo de Investigación}

Se realizó un estudio con un diseño no experimental de tipo descriptivo, analítico, prospectivo y de corte transversal con un enfoque cuantitativo.

\subsection{Participantes}

La población objetivo del estudio fue 22428 mujeres de la cuidad de Azogues en edades entre 18 y 49 años. La muestra se calculó utilizando el programa informático de datos Epidat 4.1, de acuerdo con los siguientes parámetros: a) Tamaño de la población $=22428$ mujeres, b) Proporción esperada $=10 \%, \mathrm{c}$ ) Nivel de confianza $=95 \%, \mathrm{~d}$ ) Exactitud absoluta $=1 \%$. La muestra estaba constituida por 351 mujeres que asisten a la consulta externa. El muestreo fue aleatorio simple. Los criterios de inclusión fueron los siguientes: a) mujeres azogueñas, b) edades entre 18 y 49 años, y c) aceptar participar en la investigación.

\subsection{Instrumentos}

En primer lugar, se aplicó una encuesta de las variables sociodemográficas (edad, procedencia, etnia, estado civil, ocupación y nivel de instrucción) y posteriormente, para la recolección de datos se elaboró una Escala Violencia de Género en Mujeres (EVG), el cual fue aplicado a una muestra piloto con los estudiantes $(n=80)$ de la Escuela de Enfermería Azogues. El cuestionario se tituló: de 15 a 49 años de edad que acuden al Centro de Salud $N^{\circ} 1$ de Azogues, explicándoles el motivo de estudio, confidencialidad y anonimato, recopilando datos de carácter sociodemográficos y preguntas sobre tipos de violencia, con un total de 27 preguntas (patrimonial 7, psicológica 8, física 7 y sexual 5). Se utilizó la escala de LIKERT con los siguientes ítems: muchas veces, pocas veces, una vez y nunca, la información fue tomada del Instituto Nacional de Estadísticas y Censos (INEC), elaborada por los investigadores y validada por expertos: Mgs. Maritza Galán, Mgs Luis Astudillo, Mgs. Andrés Ramírez.

\subsection{Procedimiento}

Se realizó la construcción de la escala EVG-19 para las poblaciones ecuatoriana. Primero se efectuó la lectura de cada ítem, identificando los vocablos utilizados y su significado dentro de la lengua castellana y la cultura ecuatoriana. Como segundo paso, se les solicitó a seis profesionales (dos psicólogos sociales, dos especialistas en Violencia de Género y dos literarios) que evaluaron la redacción y comprensión de los enunciados, los cuales concluirán que la redacción neutral de cada enunciado los hace entendible para la población adulta seleccionadas. En tercer lugar, se aplicó a un grupo de 80 estudiantes (mujeres), para observar cómo responden los ítems de la Escala EVG-19 
e identificar inconvenientes. Se leyó cada ítem comprobando la comprensión del enunciado y las alternativas de respuestas posibles, de esta forma también se verificó que cada persona contestar la totalidad de los ítems. Se solicitó el permiso a las autoridades de la Universidad Católica de Cuenca, para aplicar la escala EVG-19 en la ciudad de Azogues. Permitiendo el acceso a los estudiantes de la carrera de pregrado de enfermería, se entregó a cada adulto el consentimiento informado, que incluyó la solicitud de participación en el estudio y de los objetivos del estudio. Una vez obtenido el consentimiento aceptado de los adultos se procesó con el llenado de la escala EVG-19 de manera virtual. Las participantes (mujeres de la cuidad de Azogues) para el presente estudió fueron contratados a través de correo electrónico, blogs y medios sociales. El consentimiento informado se obtendrá electrónicamente antes de que, se recogieran los datos de los participantes.

\subsection{Análisis estadístico}

Se utilizó el análisis descriptivo para conocer las características sociodemográficas de la población de estudio mediante frecuencias (f) y porcentajes (\%). Posteriormente se realizó una prueba de normalidad de las variables de estudio mediante la prueba de Shapiro Wilk (W). En la que se obtuvo supuestos no paramétricos. Por lo tanto, se realizó el análisis correlacional mediante la prueba de Rho de Spearman, por ser esta prueba adecuadamente vigorosa ante el cumplimiento de supuestos no paramétricos. Los análisis se efectuaron con el programa estadístico SPSS versión 26.

\section{Resultados}

Con relación a las características sociodemográficas (Tabla 1) se obtuvieron los siguientes resultados se clasifico la edad en rangos según la Organización Mundial de la Salud (OMS) en la que consta adolescencia, adulto joven y adulto, existiendo mayor prevalencia en adulto joven con el $57.3 \%$, residiendo la mayoría en el área urbana con el $58.7 \%$, la mayor parte era de etnia mestiza que representa el $91.7 \%$.

La mayor parte de mujeres estaba soltera con el $38.7 \%$ seguida por la casada con el $32.2 \%$, un $39 \%$ son amas de casa, existiendo un $37.9 \%$ con un nivel de instrucción de secundaria completa. Cabe recalcar que en la población de estudia existe un mínimo porcentaje $0.6 \%$ tienen el cuarto nivel y el mismo porcentaje no tienen estudios.

Tabla 1: Características sociodemográficas

\begin{tabular}{|c|c|c|c|}
\hline & & $f$ & $\%$ \\
\hline \multirow{3}{*}{ Edad } & Adolescencia & 57 & 16,2 \\
\hline & Adulto joven & 201 & 57,3 \\
\hline & Adulto & 93 & 26,5 \\
\hline \multirow{4}{*}{ Procedencia } & Indígena & 24 & 6,8 \\
\hline & Mestiza & 322 & 91,7 \\
\hline & Afroecuatoriana & 3 & 0,9 \\
\hline & Otros & 2 & 0,6 \\
\hline \multirow{6}{*}{ Estado civil } & Soltera & 136 & 38,7 \\
\hline & Casada & 113 & 32,2 \\
\hline & Unión Libre & 67 & 19,1 \\
\hline & Separada & 13 & 3,7 \\
\hline & Divorciada & 18 & 5,1 \\
\hline & Viuda & 4 & 1,1 \\
\hline \multirow{5}{*}{ Ocupación } & Trabajo remunerado & 63 & 17,9 \\
\hline & Trabajo ocasional(subempleado) & 34 & 9,7 \\
\hline & Desempleada & 12 & 3,4 \\
\hline & Ama de casa & 137 & 39,0 \\
\hline & Estudiante & 105 & 29,9 \\
\hline \multirow{8}{*}{ Nivel de instrucción } & Primaria completa & 63 & 17,9 \\
\hline & Primaria incompleta & 19 & 5,4 \\
\hline & Secundaria completa & 133 & 37,9 \\
\hline & Secundaria incompleta & 95 & 27,1 \\
\hline & Tercer nivel & 37 & 10,5 \\
\hline & Cuarto nivel & 2 & 0,6 \\
\hline & Sin estudios & 2 & 0,6 \\
\hline & Total & 351 & 100 \\
\hline
\end{tabular}


En relación a los tipos de violencia se revelan datos que el $22.5 \%$ ha sufrido violencia patrimonial (el $19.7 \%$ por una ocasión y el $2.8 \%$ pocas veces), el $45.6 \%$ ha sido víctima de violencia psicológica $(36.5 \%$ una vez, $8.3 \%$ pocas veces y $0.9 \%$ muchas veces), el $20.8 \%$ presencia violencia física ( $16.8 \%$ una vez, $3.7 \%$ pocas veces y $0.3 \%$ muchas veces), según la violencia sexual $13.1 \%$ (10.3\% una vez, $2.3 \%$ pocas veces y $0.6 \%$ muchas veces).

Tabla 2: Tipos de violencia-Ausencia y Presencia de violencia

\begin{tabular}{|c|c|c|c|c|c|c|}
\hline & & $\mathrm{f}$ & $\%$ & & $\mathrm{f}$ & $\%$ \\
\hline \multirow{3}{*}{ Patrimonial } & Nunca & 272 & 77,5 & \multirow{2}{*}{ Ausencia } & \multirow{2}{*}{272} & \multirow{2}{*}{77,5} \\
\hline & Una vez & 69 & 19,7 & & & \\
\hline & Pocas veces & 10 & 2,8 & Presencia & 79 & 22,5 \\
\hline \multirow{4}{*}{ Psicológica } & Nunca & 191 & 54,4 & \multirow{2}{*}{ Ausencia } & \multirow{2}{*}{191} & \multirow{2}{*}{54,4} \\
\hline & Una vez & 128 & 36,5 & & & \\
\hline & Pocas veces & 29 & 8,3 & \multirow{2}{*}{ Presencia } & \multirow{2}{*}{160} & \multirow{2}{*}{45,6} \\
\hline & Muchas veces & 3 & 0,9 & & & \\
\hline \multirow{4}{*}{ Física } & Nunca & 278 & 79,2 & \multirow{2}{*}{ Ausencia } & \multirow{2}{*}{278} & \multirow{2}{*}{79,2} \\
\hline & Una vez & 59 & 16,8 & & & \\
\hline & Pocas veces & 13 & 3,7 & \multirow{2}{*}{ Presencia } & \multirow{2}{*}{73} & \multirow{2}{*}{20,8} \\
\hline & Muchas veces & 1 & 0,3 & & & \\
\hline \multirow{5}{*}{ Sexual } & Nunca & 305 & 86,9 & \multirow{2}{*}{ Ausencia } & \multirow{2}{*}{305} & \multirow{2}{*}{86,9} \\
\hline & Una vez & 36 & 10,3 & & & \\
\hline & Pocas veces & 8 & 2,3 & \multirow{2}{*}{ Presencia } & \multirow{2}{*}{46} & \multirow{2}{*}{13,1} \\
\hline & Muchas veces & 2 & 0,6 & & & \\
\hline & Total & 351 & 100 & Total & 351 & 100 \\
\hline
\end{tabular}

La relación entre características sociodemográficos y tipos de violencia es la siguiente: la edad tiene relación con la violencia patrimonial, psicológica y física, con un nivel de significancia positiva CC:220 >0.005, estado civil se relaciona con todos los tipos de violencia de forma positiva, nivel de instrucción se relaciona con la violencia patrimonial, psicológica y física con un nivel de significancia negativo CC:-151<0.005.Segun el análisis de esta tabla los tipos de violencia no tienen ninguna relación con la procedencia, etnia y ocupación.

Tabla 3: Correlación entre características sociodemográficos y tipos de violencia

\begin{tabular}{lcccc}
\hline & Patrimonial & Psicológica & Física & Sexual \\
\hline Edad &, $220 * *(.00)$ &, $200 * *(.00)$ &, $227 * *(.00)$ & $0,091(.08)$ \\
Procedencia & $0,011(.08)$ & $-0,046(.03)$ & $-0,006(.09)$ & $-0,064(.02)$ \\
Etnia & $0,007(.89)$ & $0,036(.49)$ & $0,016(.77)$ & $0,062(.24)$ \\
Estado civil &, $284 *(.00)$ &, $228 * *(.00)$ &, $270 * *(.00)$ &, $116^{*}(.03)$ \\
Ocupación & $0,046(.39)$ & $-0,028(.59)$ & $-0,034(.52)$ & $0,026(.62)$ \\
Nivel de instrucción &,$- 151 * *(.00)$ &,$- 133 *(.01)$ &,$- 114 *(.03)$ & $-0,005(.92)$ \\
\hline
\end{tabular}

Valor de $\mathrm{p}<.05=*$ y $\mathrm{p}<.01$

\section{Discusión}

Este estudio tiene como finalidad describir los tipos de violencia que sufren las mujeres en las relaciones de pareja en la que incluye la violencia patrimonial, psicológica, física y sexual, de forma que se conozca los factores sociodemográficos y la relación con la violencia, en mujeres que acuden a consulta externa en el Centro de Salud de Azogues.

Esta investigación se llevó a cabo con la participación de las mujeres de 15 a 49 años edad que se encontraron en la sala de espera, en donde el análisis de las características sociodemográficas dio una mayor prevalencia en la población del adulto joven, que tiene como ocupación ser ama de casa, quizás esta es una de las razones de dependencia económica de la pareja, impidiéndole romper lazos de violencia sufrida día a día en silencio.

Cano et al, en su estudio realizado en mujeres que asisten a la atención primaria de salud, en los resultados manifiesta que la gran parte de mujeres pertenecían al área urbana con el 63,8\% comparando con presente artículo el 58,7\% forman parte de la misma, el 40,2\% tenían estudios primario y secundarios $27,2 \%$, resultados similares a nuestro estudio en donde las mujeres tenían la primaria completa el $17,9 \%$ y la secundaria completa el $37,9 \%$ evidenciando una disminución del nivel de instrucción de la población de estudio en donde la mayoría solo había terminado la secundaria completa. ${ }^{16}$ 
Jaen et al. ${ }^{17}$ mencionaron resultados similares al presente estudio con relación a los tipos de abuso que concuerdan con los tipos de violencia con mayor presencia en el estudio son la violencia psicológica el $32 \%$, física $19 \%$, económica $14 \%$ y sexual con el $8,5 \%$ datos similares al presente artículo con respecto a la violencia psicológica el 45,6\%, patrimonial 22,5 , física $20,8 \%$ y sexual $13,1 \%$, el uso de preguntas cerradas facilita las mujeres a que den una respuesta concreta, ; los hallazgos de este estudio muestran que la violencia sigue existiendo en la población en donde se vulneran los derechos y se mira al mujer como un objeto sexual.

Pérez et al. ${ }^{18}$ en su estudio contra la mujer en la pareja, un estudio realizado en la atención primaria revela datos donde el $25.6 \%$ eran solteras, ocupación ama de casa un $35.3 \%$, nivel de instrucción secundaria $23.7 \%$, tipo de maltrato físico $5.4 \%$, emocional $16.9 \%$ y sexual $3.7 \%$, existe relación con respecto a nuestro estudio con los tipos de violencia, ya que en la actualidad aún existe un índice elevado de casos de violencia donde la mujer es vulnerada.

\section{Conclusión y recomendaciones}

Los resultados de la presente investigación revelan que una proporción considerable de usuarias que acuden a consulta externa sufren algún tipo de violencia. Según las características sociodemográficas existe un porcentaje elevado de población adulto joven que residen en el área urbana, etnia mestiza, estado civil solteras y escolaridad secundaria completa.

Es evidenciable los diversos tipos de violencia que padecen las mujeres que acuden a esta casa de salud, ya que la más frecuente es la psicológica, patrimonial, física y sexual. Estas situaciones dificultan a la víctima a salir de 10 . las relaciones de maltrato por situaciones diversas como sumisión, temor, falta de apoyo por parte de la familia afectando de manera significativa a la agredida y al entorno.

Razón por lo cual se considera importante intervenir de una manera inmediata y ayudar a las maltratadas para prevenir complicaciones; el personal de salud cumple un papel fundamental en este espacio, para actuar y detectar estas situaciones y detectar este problema de salud pública.

\section{Fuente de Financiamiento}

Este estudio es autofinanciado.

\section{Conflicto de Intereses}

No existen conflictos personales, profesionales, financieroso de otro tipo.

\section{Consentimiento Informado}

Los autores cuentan con el consentimiento informado de los pacientes para la investigación, la publicación del caso y sus imágenes.

\section{Referencias Bibliográficas}

1. Fernández M. Salud y Violencia de Género en América latina: Una Lectura de Género. Ministerio de Salud. 2016; $1(1)$.

2. Calvo G, Camacho R. La violencia de género: evolución, impacto y claves para su abordaje. Enfermería Global. 2014; 13(3): 15-28.

3. Marcano A, Palacios Y. Violencia de género, categorización, causas y consecuencias. Comunidad y Salud. 2017; 15(3): 73-85.

4. UNICEF. Hendel Perspectiva de género. [Online]; 2017. Disponible en: https://www.unicef.org/ argentina/sites/unicef.org.argentina/files/2018-04/com1_PerspectivaGenero_WEB.pdf.

5. Virgnolo J. Niveles de atención de prevención y atención primaria de salud. Archivo de Medicina Interna. 2011; 33(1): 11-14.

6. Antunes M, Montalbán M, Signorini H. Discurso sobre la violencia hacia la mujer en un foro virtual: Presencias del marco de género. Gaceta Sanitaria. 2013; 27(2): 111-115.

7. Yungeros A. La violencia contra las mujeres: Conceptos y Causas. Revista CastellanoManch de Ciencias Sociales. 2014; 18(1): 147-159.

8. Puente A, Ubillos S, Echenurua E, Páez D. Factores de Riesgo asociados a la violencia sufridos por la mujer en pareja: Una revisión de metaanálisis y estudios recientes. Anales psicológicas. 2016; 32(1): 295-306.

9. OMS.. Informe de la OMS destaca que la violencia contra la mujer es "Un problema de Salud global de proporciones epidémicas. [Online]; 2013. Disponible en: https://www.who.int/mediacentra/news/relaces/ 2013/violencia_against_women_2013062.

10. INEC. Compendio Estadístico. [Online]; 2014. Disponible en: https://www.ecuadorencifras.gob.ec/compendioestadistico-2016/.

11. Chávez M, Méndez A. Violencia de Género en Ecuador. Revista Publicando. 2016; 3(8): 114-115.

12. Illescas M, Tapia J, Flores E. Factores socioculturales que influyen en mujeres víctimas de violencia intrafamiliar. Revista Killkana Sociales. 2018; 2(3): 187-196.

13. Piedra J, Rosa J, Muñoz M. Intervención y Prevención de la violencia de género: Un acercamiento desde el trabajo social. Trabajo Social Global. 2018; 8(14): 195-216.

14. Lemus O, Acosta Y, Damas L. Violencia intrafamiliar contra la mujer. Revista Cubana de Enfermería. 2016; 32(1): 98-109.

15. Ruiz Pérez I, Pastor Moreno G. La violencia intrafamiliar. Un problema de salud actual. [Online].; 2020. Acceso 24 de junio de 2020. Disponible en: http: //www.observatorio.saluddecaldas.gov.co/desca/saludm/ Informe $\% 20$ evento $\% 20875 \% 20$ Violencias $\% 20 \mathrm{de} \% 20 \mathrm{~g} \%$ C3\%A9nero\%20Semestre\%20I\%202017.pdf.

16. Cano L, Berrocoso A, Arriba L, Quirós L, Alamar J, Cardo A. Prevalencia de Violencia de género en mujeres que acuden a consultas de Atención Primaria. Revista Clínica Médica Familiar. 2010; 3(1): 10-17. 
17. Jaen C, Rivera S, Amorín E, Rivera L. Violencia de Pareja en Mujeres; prevalencia y factores asociados. Acta de Investigación Psicológica. 2015; 5(3): 2224-2239.

18. Ruiz I, Plazaola J, Blanco P, González J, Ayuso P, Montero M. La violencia contra la mujer en la pareja. Un estudio en el ámbito de la Atención primaria. Gaceta Sanitaria. 2006; 20(3): 202-208.

Recibido: 9 de octubre de 2020

Aceptado: 25 de octubre de 2020

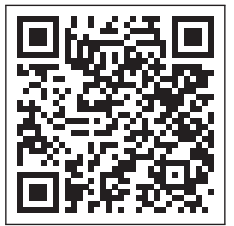


